\title{
Seismic hazard assessment using apparent stress ratio
}

\author{
LG Brown Laurentian University, Canada \\ MR Hudyma Laurentian University, Canada \\ P Turcotte Agnico Eagle Mines Ltd, Canada
}

\begin{abstract}
Seismically active operations require special considerations for design and mining practices in order to mitigate the risk associated with large and potentially damaging seismic events. When detailed knowledge of the local rock mass conditions and the historical seismic response is considered during mine design, costly rehabilitation and time delays can be minimised.

High apparent stress is an indicator of increasing stress within the rock mass. Apparent stress ratio (ASR) is a new and innovative way of quantifying apparent stress for a given seismic population. A high ASR value suggests that local stress conditions are high and increasing. Analysis of seismic event data from LaRonde Mine, Canada, suggests that the ASR is strongly correlated with the occurrence of large events - high seismic hazard. This paper presents the use of ASR as a precursory trend to large seismic events for both intermediate and long term seismic hazard. Practical examples are provided from LaRonde Mine.
\end{abstract}

\section{Introduction}

Seismic monitoring and analysis are critical components to hazard assessment, particularly in deep and high stress mining environments such as Agnico Eagle's LaRonde Mine, shown in Figure 1. LaRonde is a Canadian operation located in northern Quebec, approximately $650 \mathrm{~km}$ northwest of Montreal near the town of Preissac. It is a world-class Au-Ag-Cu-Zn massive sulphide lens complex with over 3.4 million ounces of gold in proven and probable reserves (Agnico Eagle Mines Limited 2015). Current mining extends more than 2,930 metres below the surface, producing approximately 6,150 tonnes per day (Agnico Eagle Mines Limited 2015). Bulk mining at such depth has resulted in a history of seismic activity at LaRonde, which has been recorded since 2003.

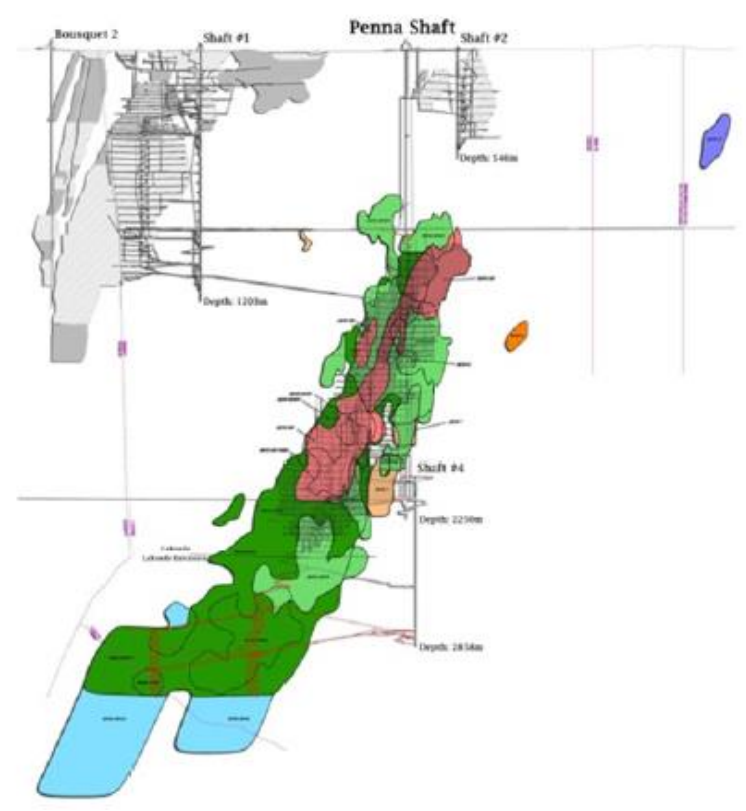

Figure 1 Long section of LaRonde Mine (Turcotte 2014) 


\subsection{Apparent stress}

Many forms of seismic analysis use source parameters to determine areas of elevated seismic hazard. Apparent stress $\left(\sigma_{a}\right)$ is a measure of stress change at a seismic source. It was originally defined by Wyss and Brune (1968) as:

$$
\sigma_{\mathrm{a}}=\mu(\mathrm{E} / \mathrm{Mo})
$$

where:

$$
\begin{array}{ll}
\sigma_{\mathrm{a}} & =\text { apparent stress }\left(\mathrm{Pa}=\mathrm{N} / \mathrm{m}^{2}\right) . \\
\mu & =\text { shear modulus of rigidity of the source material }\left(\mathrm{N} / \mathrm{m}^{2}\right) . \\
\mathrm{E} & =\text { seismic energy }(\text { Joules }=\mathrm{Nm}) . \\
\text { Mo } & =\text { seismic moment }(\mathrm{Nm}) .
\end{array}
$$

High apparent stress values are indicative of high and increasing stress conditions within the rock mass (Mendecki 1993). Determining what constitutes high apparent stress is difficult and can vary greatly with changes in geological and stress conditions. Current methods that utilise apparent stress for hazard assessment, such as apparent stress time history charts (Young 2012), require the selection of an arbitrary threshold value to represent high apparent stress. There are disadvantages in using this approach, as it needs to be customised to represent changes in local rock mass conditions. What may be high apparent stress for one mine or a region within a mine, may be moderate or even relatively low apparent stress for another mine or region. Figure 2 depicts cumulative apparent stress distributions for seismic activity at LaRonde Mine on four levels of varying depth.

A clear increase in apparent stress values is evident as the depth of mining increases. The selection of a single threshold value to represent high apparent stress cannot be reliably applied across multiple mining levels. In order to overcome this limitation, this paper presents the use of an ASR analysis technique. This allows the seismic response to local stress and geological conditions to be compared relative to other data within the same population at LaRonde.

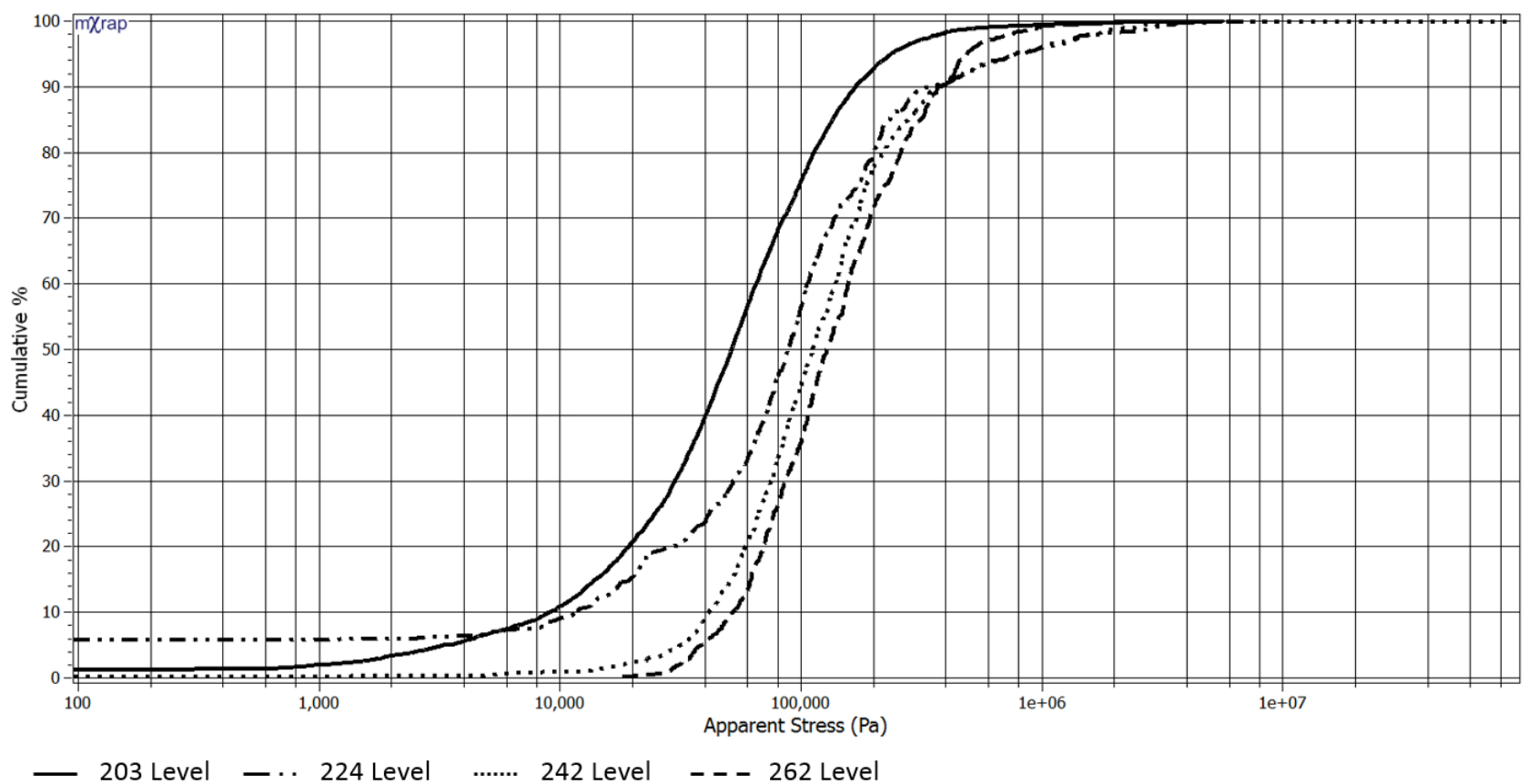

Figure 2 Apparent stress cumulative distribution for the 203, 224, 242, and 262 levels at LaRonde (Brown 2015) 


\section{Apparent stress ratio}

The intent of ASR is to identify increasing apparent stress within a seismic population as a proxy for increasing stress conditions within a rock mass. For any given population the ASR value is calculated as the ratio of the apparent stress 80th percentile to the 20th percentile for a cumulative distribution of the population.

$$
\operatorname{ASR}=\frac{\sigma_{\mathrm{a} 80}}{\sigma_{\mathrm{a} 20}}
$$

where:

$$
\begin{aligned}
& \sigma_{\mathrm{a} 80}=\text { apparent stress 80th percentile. } \\
& \sigma_{\mathrm{a} 20}=\text { apparent stress 20th percentile. }
\end{aligned}
$$

A high ASR suggests that the apparent stress is relatively high within the population and that local stress conditions are high and increasing within the rock mass. A low ASR suggests that the apparent stress is relatively constant within a population and that local stress conditions are constant or decreasing. By using the $20^{\text {th }}$ and $80^{\text {th }}$ percentile values, the extremities of the distribution can be considered while minimising the impact of a few anomalously high or low apparent stress events. The concept of apparent stress ratio is similar to the uniformity co-efficient in soil mechanics, which is used to quantify variations in a particle size distribution.

An implicit assumption is that increasing stress conditions within a rock mass lead to rock mass instability and the occurrence of large seismic events. For seismic source mechanisms not related to increasing stress, apparent stress may be unrelated to the occurrence of large seismic events. Determining an uncharacteristically high ASR value to associate with elevated seismic hazard may depend on a number of factors. Potentially one of the most influential factors is the preceding time period over which the ASR value is calculated. Variations in the time periods considered for calculation could provide insight into short, intermediate, and long-term seismic hazard.

\subsection{Time periods for seismic hazard assessment using ASR}

Trends in seismic data can be analysed over varying time periods. This paper proposes the use of ASR to assess intermediate and long-term seismic hazard. When a time period is selected, the ASR value for each event is calculated from only the preceding events contained within the specified time window. For example, assessing hazard using a window of one year would result in an ASR that only considers seismicity occurring within the preceding 365 days.

Van Aswegen (2005) suggested varying time periods for seismic hazard assessment. Short-term seismic hazard refers to hours and days, medium term refers to a monthly planning cycle, and long term refers to a time span that allows for changes in mine design in the order of a year (van Aswegan 2005). For application of this methodology to LaRonde, intermediate term is defined as a typical planning period of three months and long term as one year. ASR values can vary substantially based on the selected time period. Short time periods, in the order of days to weeks, are very limited in their application as populations with relatively low event rates over extended periods of time do not allow for substantial stress change to be observed.

\section{Seismic monitoring at LaRonde}

The LaRonde Engineering Seismology Group (ESG) seismic monitoring hardware has gone through many upgrades since its implementation in 2003. The most influential change being a conversion from a Hyperion to a Paladin based system in late 2008. Prior to 2008, the sensitivity of the Hyperion seismic monitoring system was $\mathrm{M}_{\mathrm{L}}=-1.5$. The current microseismic monitoring system covers all active areas of the mine with 84 sensors, which includes seven triaxial accelerometers and 77 uniaxial accelerometers, with a sensitivity of approximately $\mathrm{M}_{\mathrm{L}}=-2.1$. 
A macroseismic regional seismic network (RSN) consisting of a single $4.5 \mathrm{~Hz}$ geophone located on surface was implemented in 2006. The purpose of this system is to provide accurate magnitudes for large, low frequency events occurring at LaRonde. Additional geophones were integrated into the network during 2012. Magnitudes after this date are typically calculated using an average of at least two sensors. Currently, the macroseismic monitoring system consists of five surface geophones. The sensors are located at distances of 2 to $15 \mathrm{~km}$ from the mine.

\subsection{Magnitude scales at LaRonde}

All magnitude scales have a logarithmic basis which allows for a large range of event size to be expressed in a short range of numbers. There are three main scales currently in use at LaRonde: Nuttli, Richter and a local magnitude. Each of these magnitude scales are generated by independent monitoring systems.

Large events that are recorded by Natural Resources Canada (NRcan) are reported with a Nuttli magnitude. This magnitude scale was developed by Nuttli (1973) specifically for small to moderate sized earthquakes in Eastern Canada. A Nuttli magnitude is 0.3 to 0.6 units higher than a Richter magnitude for the same event (Plouffe 1992).

Richter magnitudes are calculated for large events recorded by the regional macroseismic network. Richter is a commonly recognised scale for reporting seismic event magnitude. It was developed by Charles Richter (1935) for describing the size of earthquakes in California. Magnitude values are generated using the amplitude of the secondary seismic wave and the location of the instrumentation relative to the seismic source.

A local magnitude value is calculated for all events recorded by the underground ESG microseismic monitoring system at LaRonde. This scale is well suited for analysis as it provides a consistent and accurate representation for the small events and most large mining-induced seismic events recorded.

Based on data recorded at LaRonde, relations for the three magnitude scales are presented in Table 1. They are based on local, Richter and Nuttli magnitudes calculated by the ESG microseismic system, regional macroseismic system, and NRCan respectively. Future reference to event magnitude will refer to LaRonde's local magnitude scale.

Table 1 Magnitude conversion relations between local, Richter and Nuttli magnitude scales for LaRonde Mine

\begin{tabular}{ccc}
\hline Magnitude scales & Relation & Number of events \\
\hline RSN Richter and NRCan Nuttli & NRCan Nuttli = RSN Richter + 0.3 & 94 \\
RSN Richter and ESG Local & RSN Richter = ESG Local + 1.0 & 159 \\
\hline
\end{tabular}

Seismic events with a Richter magnitude greater than or equal to 1 are considered to be large and potentially damaging (Butler 1997). For LaRonde, this corresponds to a local magnitude greater than or equal to zero. Populations containing large seismic events are considered to represent areas of high seismic hazard.

\subsection{LaRonde seismic data}

In order to eliminate any bias due to changes to the seismic monitoring system, a minimum system sensitivity at LaRonde of $M_{L}=-1.5$ has been used as a lower bound. This value also reduces the influence of mining process events. Mining process events comprise the vast majority of most microseismic databases. They are small events (typically $M_{L}<-1$ ), resulting directly from stress fracturing caused by mining processes (i.e. blasting). While they are an excellent indicator of when and where mining activities are taking place, they are not necessarily directly correlated to areas of elevated seismic hazard. Figure 3 shows a cumulative distribution of apparent stress values of all events $M_{L} \geq-1.5$ within a large area of interest at LaRonde Mine. It is evident from Figure 3 that apparent stress is magnitude (scale) dependent. This ensures 
that any analysis technique correlating high hazard with large apparent stress values will incorporate most of the large magnitude seismic events.

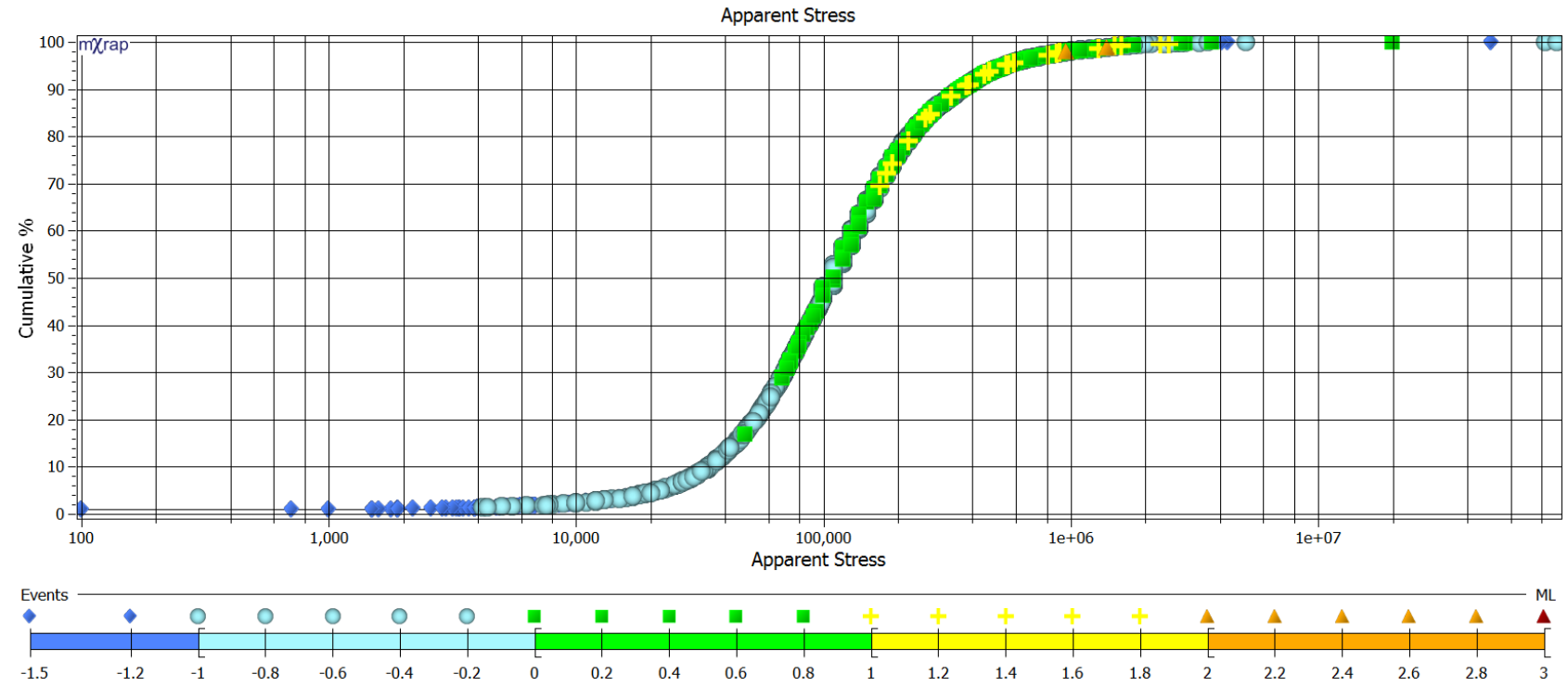

Figure 3 Apparent stress cumulative distribution for all events $M_{L} \geq-1.5$ for a large area of interest at LaRonde Mine

\section{$4 \quad$ Apparent stress ratio as an analysis tool}

Seismic analysis tools are used to gain insight into the local seismic response to mining. Selecting an appropriate time period for hazard assessment is a crucial component to effectively using ASR as an analysis tool. As stress increases within a rock mass, the individual apparent stress value of seismic events increase. If stress is capable of building quickly within a rock mass, resulting in substantial seismicity over short time periods, short-term ASR calculations may be meaningful. For most bulk mining operations however, hazard assessment spanning days may not adequately reflect stress changes in the rock mass. A medium term time period equating to a standard planning period (three months) is more likely sufficient to provide insight into changing stress conditions. An effective way of visually presenting and analysing ASR values is on an apparent stress ratio time history chart (ASRTH).

\subsection{Apparent stress ratio time history charts}

Apparent stress ratio time history charts present a variety of information about a seismic population in a single chart. Events are plotted along the $x$-axis in chronological order, with the primary $y$-axis representing event magnitude. The secondary $y$-axis is used to display ASR values, and events are coloured according to apparent stress. Figure 4 is an example of an ASRTH chart for a high hazard seismic population located in the footwall of the 242 Level at LaRonde. ASR values are calculated based on long term seismic hazard using the preceding year of seismic data. 


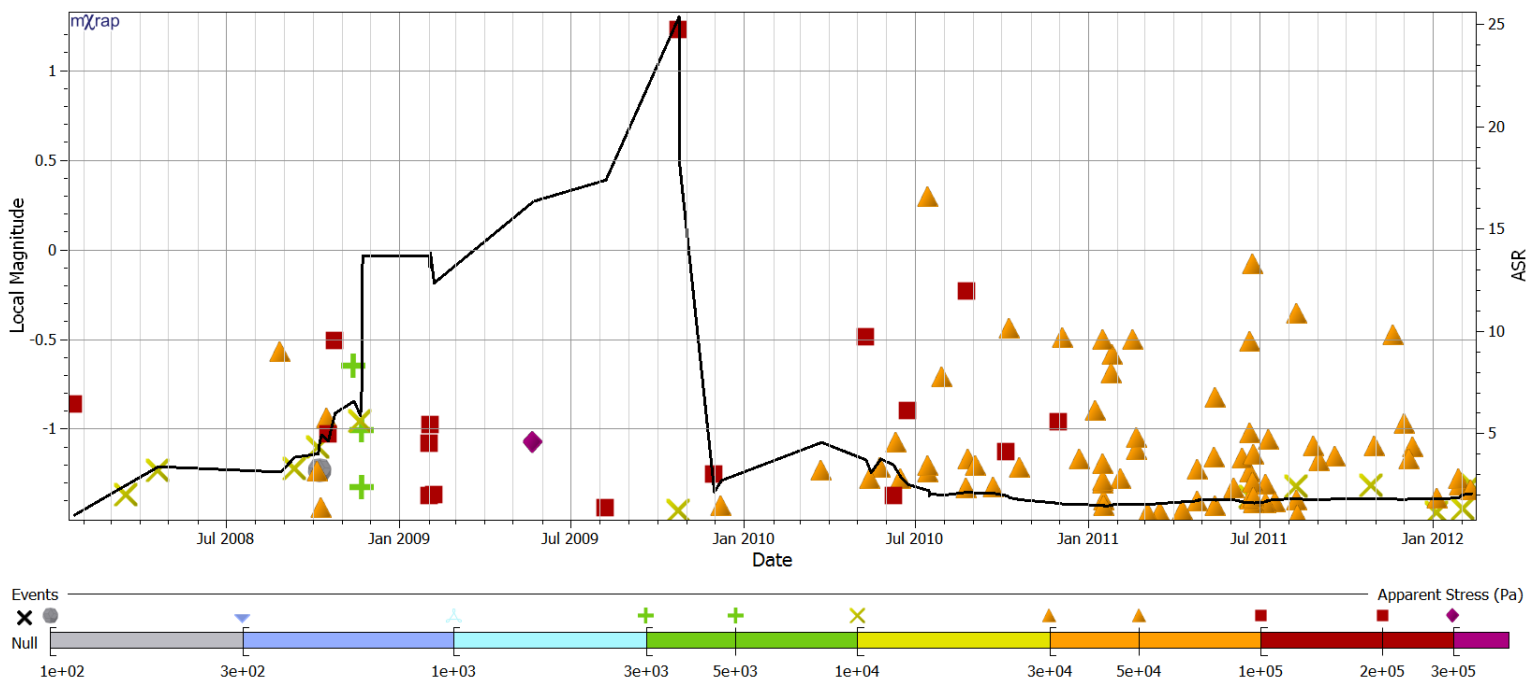

Figure 4 Apparent stress ratio time history chart for a high hazard seismic population located in the footwall of the 242 level at LaRonde Mine. ASR values are calculated for long term seismic hazard assessment, considering all events within the preceding year

At the beginning of the population life shown in Figure 4, apparent stress values of seismic events are relatively low reflected by ASR values less than 5 . As time progresses the apparent stress values begin to increase (February 2009), generating large ASR values, in excess of 10 . Although the magnitude values of these events are relatively low, the ASR values correspond to increasing stress conditions and potentially high seismic hazard. A large seismic event $\left(\mathrm{M}_{\mathrm{L}}=1.2\right)$ occurs in October 2009. Following the large event, ASR decreases significantly. There is very little variation in apparent stress values of the seismic events - even as event frequency increases. ASR values remain slightly elevated until the middle of July 2010, when there is a second large event $\left(M_{L}=0.3\right)$. ASR values then fall to levels indicative of low seismic hazard $(A S R<2)$ for the remainder of the population life.

Figure 5 is an example of an ASRTH chart for a high hazard seismic population located in the footwall of the 252 level at LaRonde. ASR values are calculated based on medium term seismic hazard - using the preceding three months of seismic data. As with all seismic parameters based analysis tools used over varying time periods, an increased measure of volatility can be seen in the ASR, as the time period is decreased.
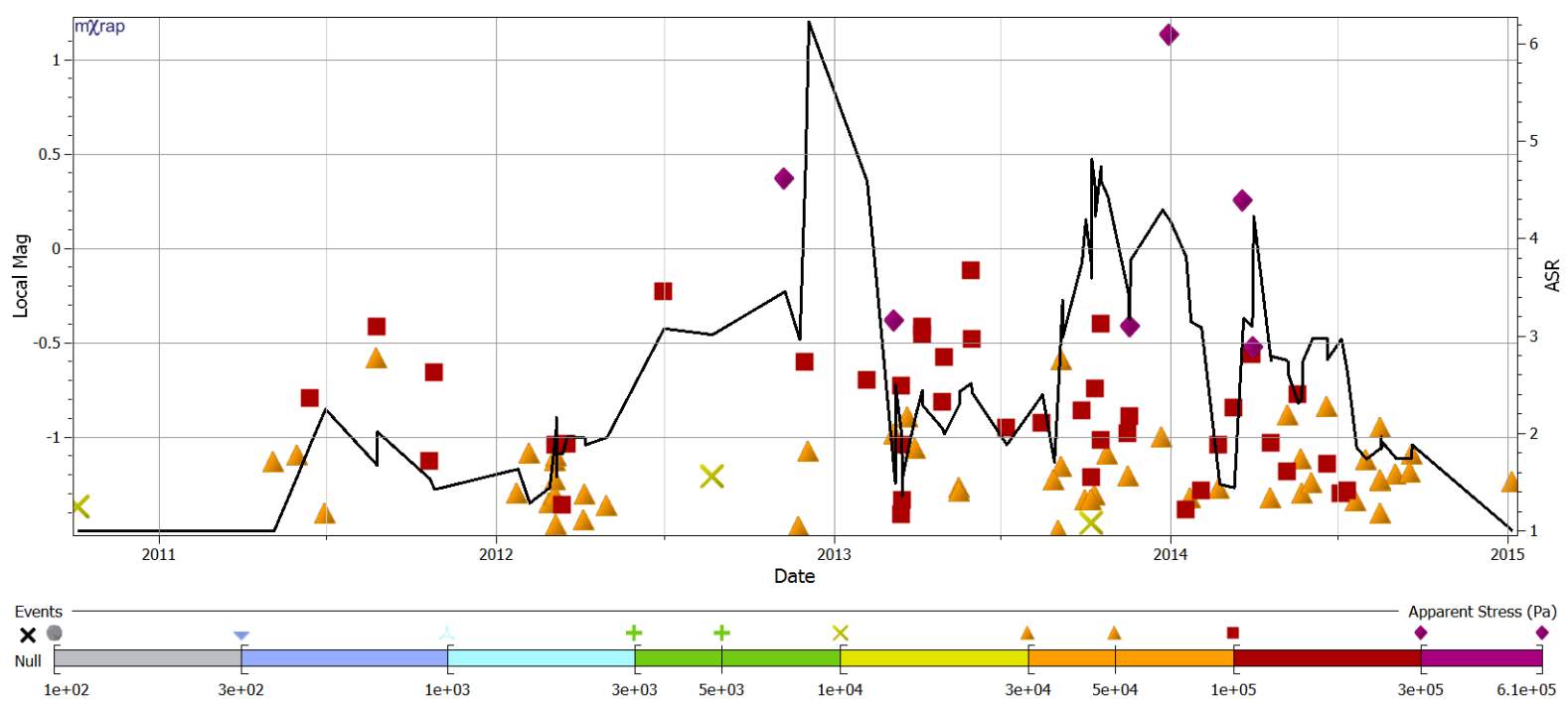

Figure 5 Apparent stress time history chart for a high hazard seismic population located in the footwall of the 252 level at LaRonde Mine. ASR values are calculated for medium term seismic hazard assessment, considering all events within the preceding three months 
Comparing Figure 4 to Figure 5, the same general trends in ASR are observed. Prior to the occurrence of the first large event $\left(M_{L}=0.4\right)$ in the population in November 2012, the ASR value is greater than three, indicative of high seismic hazard. A period of low to moderate seismic hazard is observed between March and September 2013. ASR values climb prior to the occurrence of the largest seismic event within the population $\left(M_{L}=1.1\right)$ in December 2013.

In contrast to the previous examples, Figure 6 is an example of an ASRTH chart for a low hazard seismic population located west of the ramp on the 230 level at LaRonde. ASR values are calculated based on long term seismic hazard - using the preceding year of seismic data. The majority of low hazard seismic populations at LaRonde consist nearly entirely of mining process events (typically $M_{L}<-1$ ). By employing a lower bound of $M_{L}=-1.5$ for seismic data considered in this analysis, low seismic hazard populations contain very few seismic events.

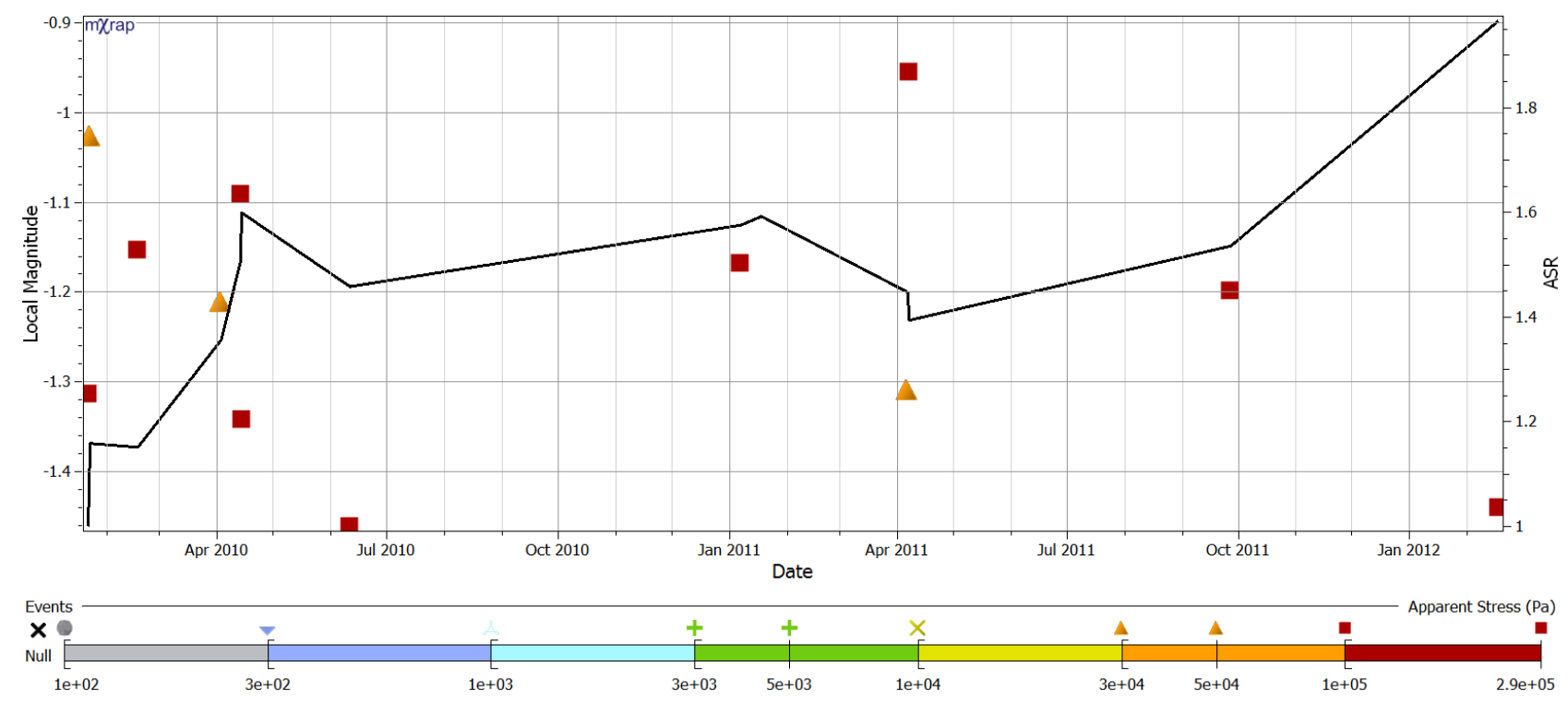

Figure 6 Apparent stress time history chart for a low hazard seismic population located west of the ramp on the 230 level at LaRonde Mine. ASR values are calculated for long term seismic hazard assessment, considering all events within the preceding year

Relative to the high hazard populations in Figures 4 and 5, ASR values for this seismic population are very low. Throughout the entire population life ASR values do not exceed two. No large variations in apparent stress are present throughout the population and, as a result, ASR values remain relatively low.

Although not shown in this paper, the use of medium and long term seismic hazard assessment produce similar results when used for the same population. This is an important component to a reliable and universally applicable seismic analysis technique. A disadvantage to using extended time periods for seismic hazard assessment is the prolonged impact from previous seismicity that may no longer be relevant to the current stress condition in the rock mass. Long term (one year) ASR values may be the best indicator of long term trends in seismic hazard, but can be influenced by old seismic events for extended periods of time. An advantage to longer time periods and consequently larger seismic populations is a reduced influence of small quantities of anomalous seismic activity. Longer time periods allow for trends to be seen in long term, gradual increase of stress within a rock mass.

\section{$5 \quad$ Large scale application of ASR at LaRonde}

The majority of ore at LaRonde is extracted using transverse open stoping with a primary/secondary pyramid mining sequence. Typical stope sizes are $30 \mathrm{~m}$ high, with widths for primary and secondary stopes of 13.5 and $16.5 \mathrm{~m}$ respectively (Mercier-Langevin 2010). Stope thickness is equivalent to the width of the orebody (to a maximum of $40 \mathrm{~m}$ ). Select areas, such as the narrow extremities, are mined using a 
longitudinal retreat open stoping mining method. Paste fill or cemented rockfill is employed for backfilling of primary stopes, while secondary stopes are backfilled using dry rockfill (Mercier-Langevin 2010).

In order to investigate the applicability of ASR as an analysis tool over a large region, 50 sample populations were selected within an area of interest at LaRonde. Populations were chosen to represent high, moderate, and low seismic hazard. Test populations refer to those that contain large seismic events and are consequently considered to reflect high seismic hazard. Within these populations, an abnormal seismic response to mining activities is expected. Control populations refer to those that do not contain large seismic events. They represent both moderate and low seismic hazard and often reflect a normal seismic response to mining activities.

\subsection{Analysis of test populations}

Test populations were generated from large seismic events located near mine workings. Selected events were used as a central point and populations were created using a $30 \mathrm{~m}$ search radius. The test populations contain 93\% (27 out of 29) of the largest events in the area of interest within $50 \mathrm{~m}$ of an excavation. The two large events not included contain only a single other event within the $30 \mathrm{~m}$ search radius and were, therefore, deemed as unable to be identified using precursory trends. Within the area of interest, $38 \%$ (55 out of 146) of all events $M_{L} \geq 0$ located within $50 \mathrm{~m}$ of an excavation are contained in the test populations.

For forecasting purposes, the ASR values preceding a large event are analysed. Figure 7 is a cumulative distribution of ASR values preceding large seismic events using medium and long term seismic hazard assessment.

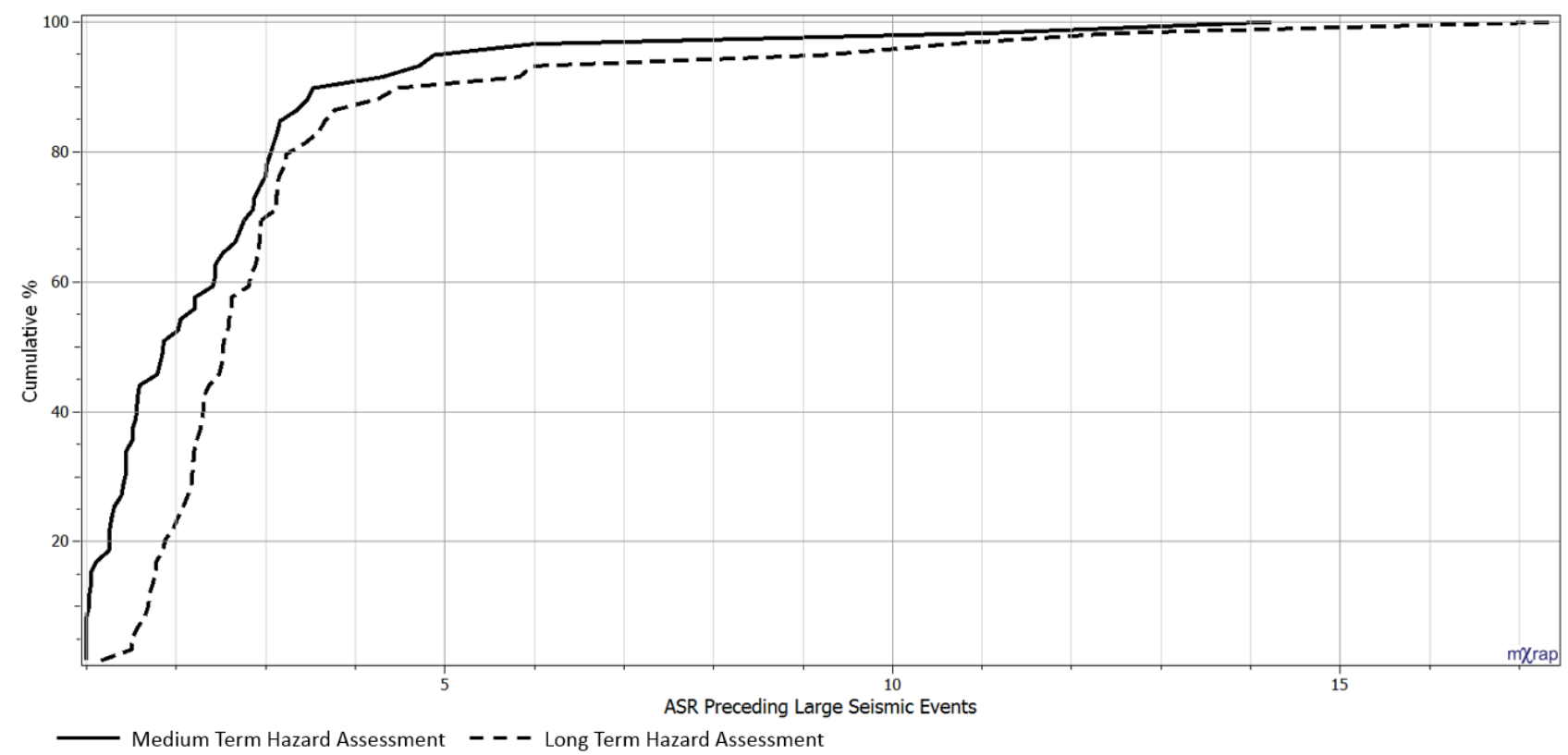

Figure 7 Cumulative distribution for ASR values preceding all large seismic events $\left(M_{L} \geq 0\right)$ contained within test populations at LaRonde Mine. ASR values calculated using medium and long term seismic hazard assessment are shown

As previously identified in the example populations, ASR values less than two are indicative of low seismic hazard. Using medium term seismic hazard assessment, approximately $50 \%$ of large events have a preceding ASR greater than two. Using long-term seismic hazard assessment, approximately $80 \%$ of large events have a preceding ASR greater than two. 


\subsection{Analysis of control populations}

Control populations do not contain large seismic events and represent moderate to low seismic hazard. In order to ensure there was no bias in the selection of central points for generating control populations, a grid was placed over the entire area of interest. An equally spaced grid of $50 \mathrm{~m}$ minimises the overlap of seismicity in populations generated from adjacent grid points. Each grid point was used as a central point to generate a seismic population using a $30 \mathrm{~m}$ search radius. Any populations containing events $M_{L} \geq 0$ or located farther than $50 \mathrm{~m}$ from excavations were eliminated, thus ensuring that the populations have no large events, i.e. the populations represent areas of low seismic hazard. The 25 grid points with the largest number of seismic events within the search radius were selected as central points to generate control populations. In cases where adjacent grid points were both selected as control populations, the one with the lower number of events was eliminated. This ensures the control populations are representative of the entire area of interest.

As previously discussed, control populations represent both moderate and low seismic hazard. Over time, moderate hazard seismic populations vary between low and high seismic hazard and therefore tend to have higher ASR values. Figure 8 is a cumulative distribution considering only the peak (largest) ASR values for all low hazard control populations using medium and long term seismic hazard assessment.

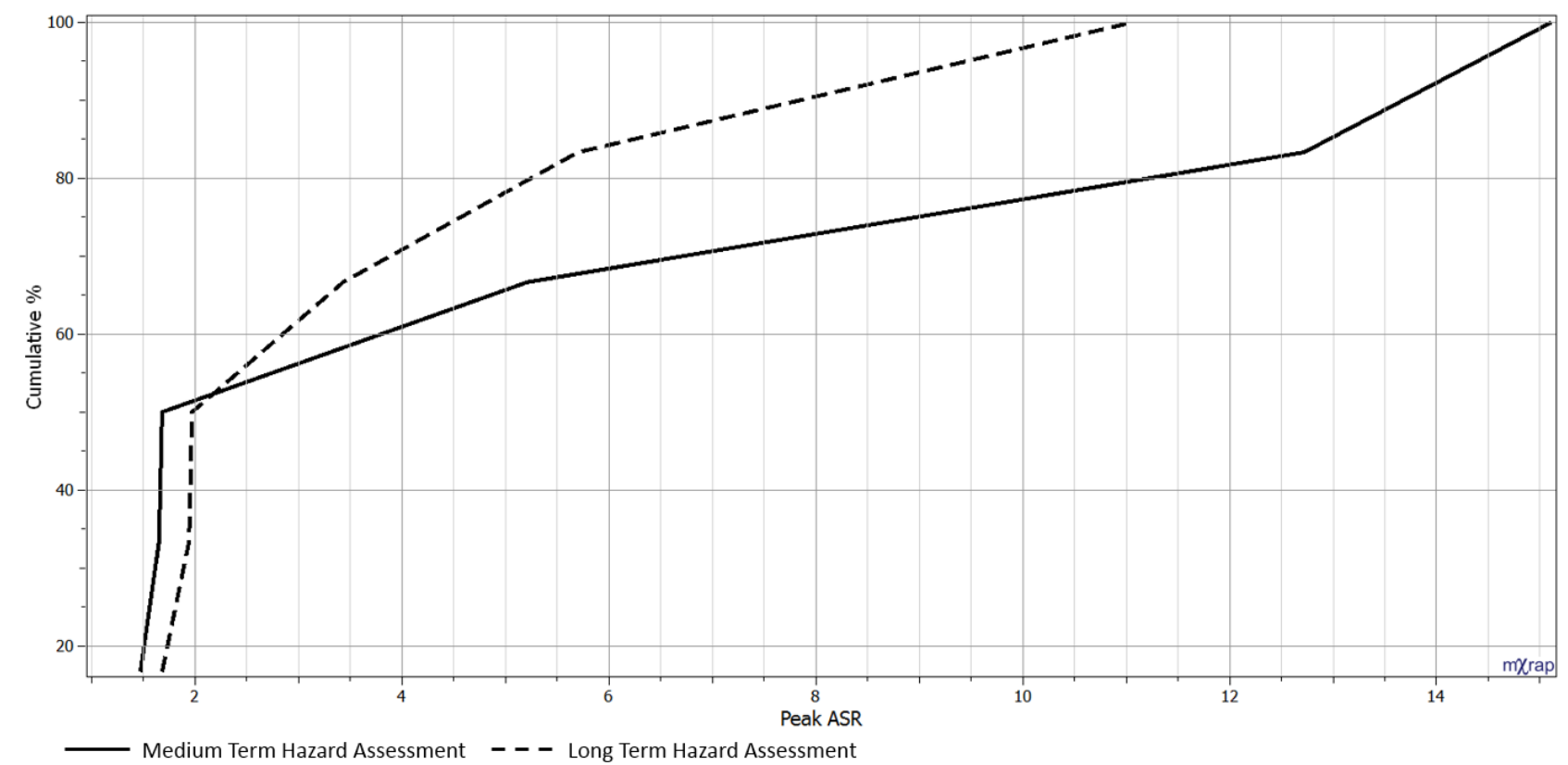

Figure 8 Cumulative distribution for peak ASR values of low hazard control populations at LaRonde Mine. ASR values calculated using medium and long term seismic hazard assessment are shown

Using a medium term seismic hazard assessment, 50\% of low hazard control populations have peak ASR values less than approximately 1.7. Using long term seismic hazard assessment, 50\% of peak ASR values are less than two. Populations that possess peak ASR values indicative of moderate to high seismic hazard $(A S R>2)$ are commonly influenced by nearby blasting, as it induces rock mass failure and stress redistribution. Time frames in which ASR values exceed two are short and typically a direct result of small quantities of events with anomalously high apparent stress values.

\section{ASR hazard mapping at LaRonde}

Using consecutive points along mine excavations and a $30 \mathrm{~m}$ search radius, an ASR hazard map can be generated. Figure 9 is a perspective side view of an ASR long term seismic hazard map highlighting three levels (248, 252 and 255) at LaRonde Mine. It was generated based on long term seismic hazard assessment and therefore only considers seismicity occurring within the previous year. 

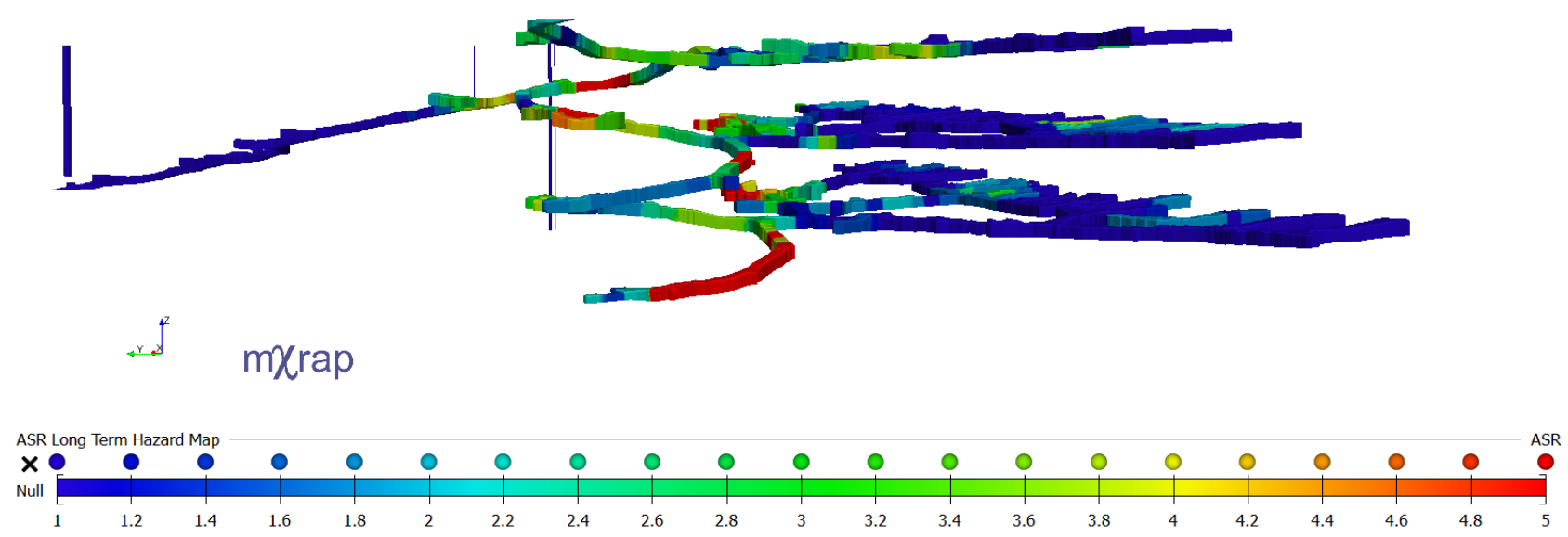

Figure 9 Perspective side view of ASR long term seismic hazard map looking approximately southeast. Levels shown are 248, 252, and 255 at LaRonde Mine

The ASR long term hazard map correlates well to the historical seismic response at LaRonde Mine. Due to varying stress, rock mass and geological conditions, areas of elevated seismic hazard at LaRonde are typically concentrated in the footwall - this is reflected in the location of the large seismic events (ML $\geq 0)$. Figure 10 is a plan view of the 248 level from the ASR long-term hazard map shown in Figure 9. All of the large events occurring near the level are shown. Not all of these events are considered in ASR calculations, as the majority have occurred prior to the preceding year.

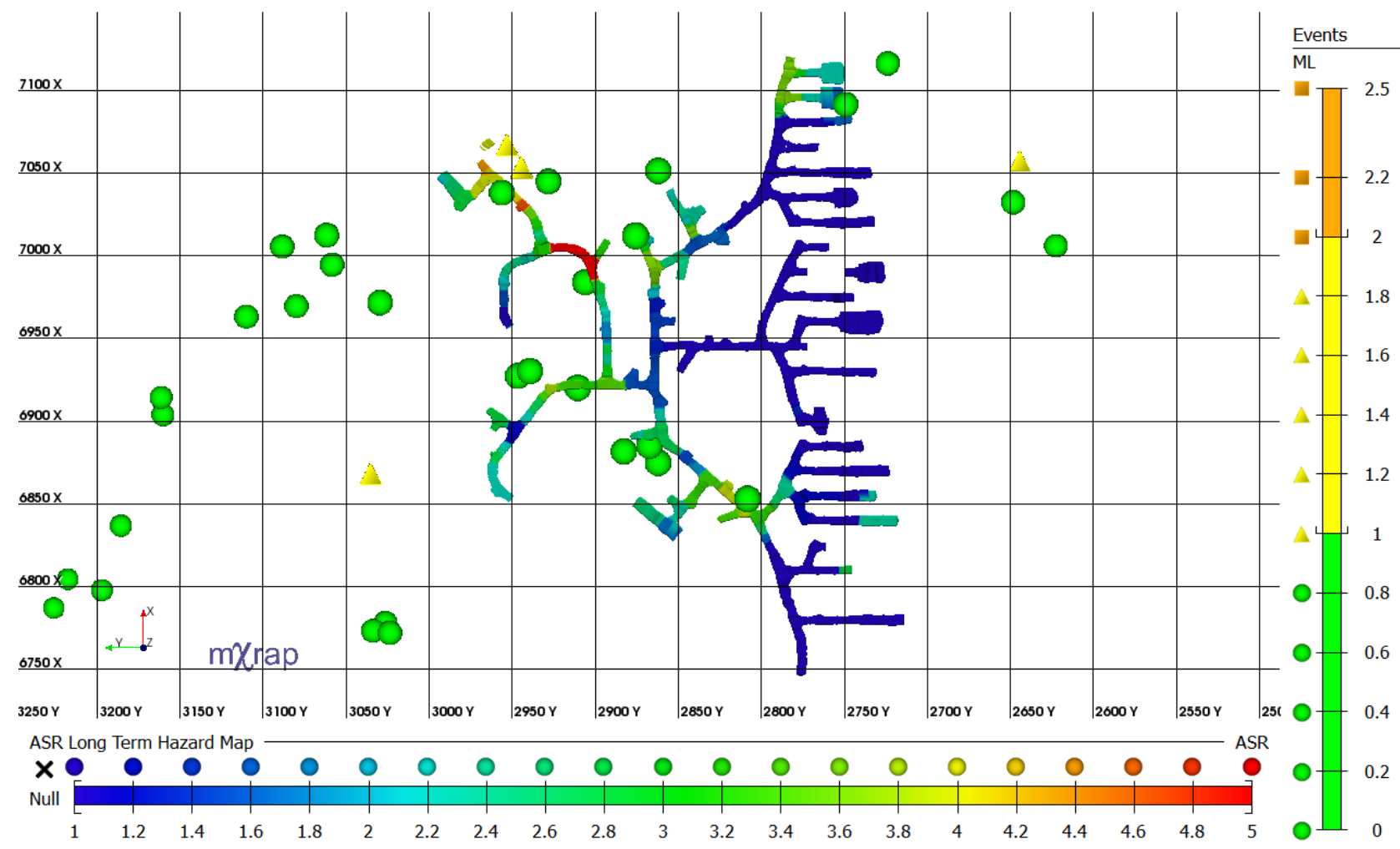

Figure 10 Plan view of the 248 level from the ASR long-term seismic hazard map for LaRonde Mine shown in Figure 9

Nearly all areas of the ASR hazard map, in the proximity of large seismic events, shows an elevated seismic hazard (ASR > 2). This is particularly significant as most of the events shown have not occurred within the preceding year and, therefore, no longer have any influence on the local ASR calculation. 
Figure 11 is a plan view of the 255 level from the ASR long-term hazard map shown in Figure 9. All of the large events occurring near the level are shown. Not all of these events are considered in ASR calculation as the majority have occurred prior to the preceding year.

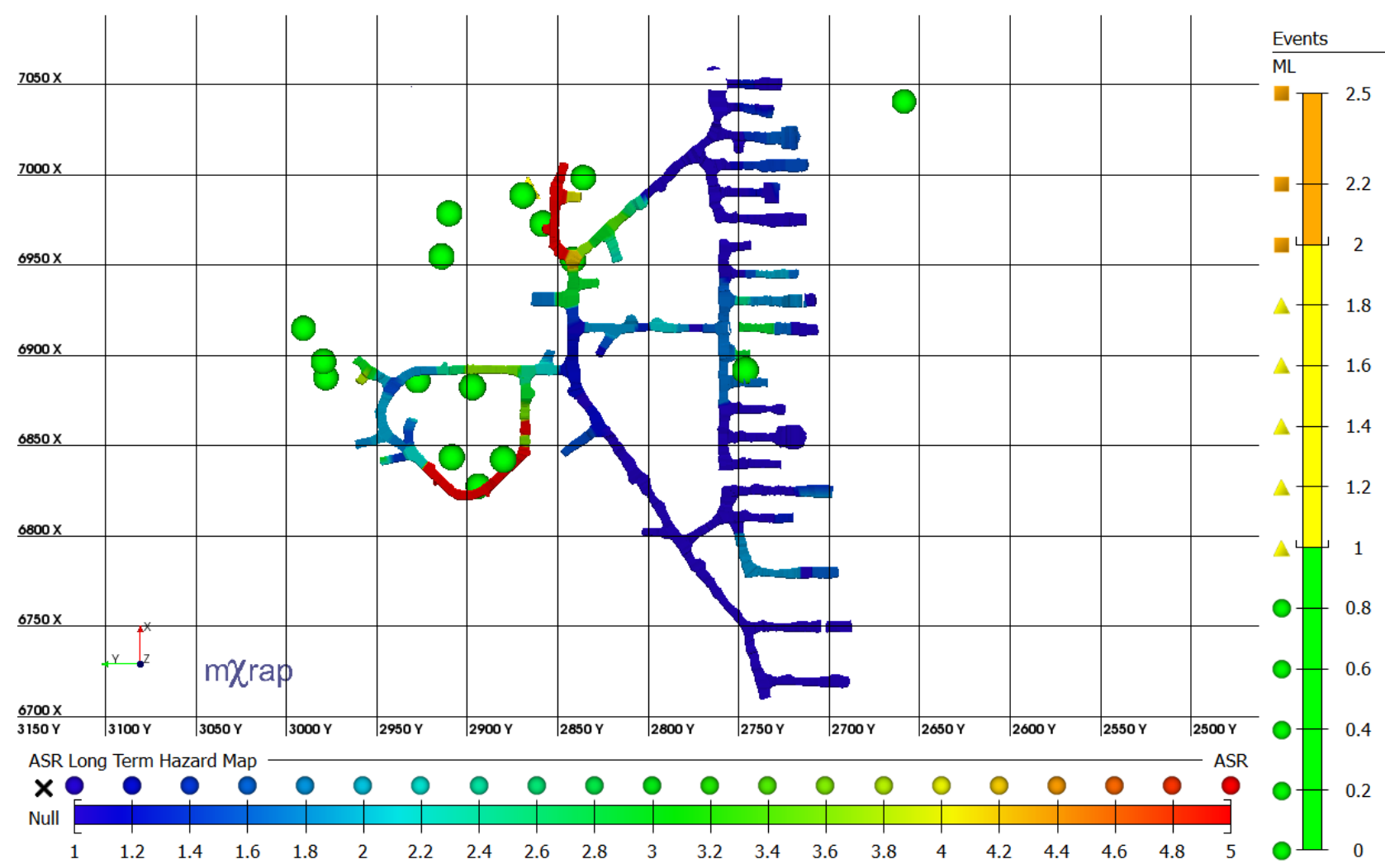

Figure 11 Plan view of the 255 level from the ASR long term seismic hazard map for LaRonde Mine shown in Figure 8

Similar to the 248 level shown in Figure 10, the areas of elevated hazard for the 255 level are concentrated in the footwall. The majority of areas in the proximity of large seismic events have ASR values indicative of elevated seismic hazard. Areas of concentrated large seismic events have ASR values indicative of very high seismic hazard (ASR > 5).

\section{Conclusion and summary}

Apparent stress ratio was found a useful seismic analysis technique at LaRonde Mine that can be applied over a variety of time frames. All of the analysis of ASR at LaRonde was completed using the mXrap software provided by the Australian Centre for Geomechanics (Harris \& Wesseloo 2015). Unlike previous analysis techniques that primarily consider apparent stress, this methodology does not require the selection of threshold values. By employing a relative ratio, all apparent stress measurements are considered in relation to those contained within the same seismic population. This eliminates the bias introduced to analysis by thresholds values that serve to quantify what is considered high and abnormal apparent stress.

\section{Acknowledgement}

This research is supported by Agnico Eagle's LaRonde Mine, the Goodman School of Mines, the Natural Sciences and Engineering Research Council, and the Canadian Engineering Memorial Foundation. A special acknowledgment is due to the ACG, specifically the mXrap team, for their much appreciated time and guidance throughout this research. 


\section{References}

Agnico Eagle Mines Limited 2015, LaRonde, Agnico Eagle Mines Limited, viewed 17 May 2015, http://www.agnicoeagle.com/en/ Operations/Northern-Operations/LaRonde/Pages/default.aspx

Brown, LG 2015, 'Seismic hazard evaluation using apparent stress ratio for mining-induced seismic events' Masters thesis, Laurentian University.

Butler, AG 1997, 'Space-time clustering of potentially damaging seismic events and seismic viscosity in Western Deep Levels East and West Mines', in SJ Gibowicz \& S Lasocki (eds), Rockbursts and Seismicity in Mines, Balkema, Rotterdam, pp. 89-93.

Harris, PC \& Wesseloo, J 2015, mXrap v5 software, Australian Centre for Geomechanics, The University of Western Australia, http://mxrap.com/

Mendecki, AJ 1993, 'Real time quantitative seismology in mines', in RP Young (ed.), Proceedings of the Third International Symposium on Rockbursts and Seismicity in Mines, Balkema, Rotterdam, pp. 287-295.

Mercier-Langevin, F 2010, 'LaRonde Extension - mine design at three kilometres', in M Van Sint Jan \& Y Povtin (eds), Proceedings of the Fifth International Seminar on Deep and High Stress Mining (Deep Mining 2010), Australian Centre for Geomechanics, Perth, pp. 3-15.

Nuttli, OW 1973, 'Seismic wave attenuation and magnitude relations for Eastern North America', Journal of Geophysical Research, vol. 78, pp. 876-885.

Plouffe, M 1992, Preliminary local magnitude scales for mining-induced seismicity at some mines in the Sudbury Basin, Mining Research Laboratories, Canada.

Richter, CF 1935, 'An instrumental earthquake magnitude scale', Bulletin of the Seismological Society of America, vol. 25, pp. 1-32.

Turcotte, P 2014, 'Practical applications of a rockburst database to ground support design at LaRonde Mine', in M Hudyma \& Y Potvin (eds), Proceedings of the Seventh International Seminar on Deep and High Stress Mining (Deep Mining 2014), Australian Centre for Geomechanics, Sudbury, pp. 79-92.

Van Aswegen, G 2005, 'Routine seismic hazard assessment in some South African mines', in Y Potvin \& M Hudyma (eds), Proceedings of the Sixth International Symposium on Rockbursts and Seismicity in Mines (RaSiM6), Australian Centre for Geomechanics, Perth, pp. 437-444.

Wyss, M \& Brune, JN 1968, 'Seismic moment, stress and source dimensions for earthquakes in the California-Nevada region', Journal of Geophysical Research, vol. 73, pp. 4681-4694.

Young, D 2012, Energy variations in mining-induced seismic events using apparent stress' Masters thesis, Laurentian University. 